PROCEEDINGS OF THE

AMERICAN MATHEMATICAL SOCIETY

Volume 133, Number 3, Pages 827-834

S 0002-9939(04)07625-7

Article electronically published on September 29, 2004

\title{
A CHARACTER OF THE GRADIENT ESTIMATE FOR DIFFUSION SEMIGROUPS
}

\author{
FENG-YU WANG
}

(Communicated by Claudia M. Neuhauser)

\begin{abstract}
Let $P_{t}$ be the semigroup of the diffusion process generated by $L:=\sum_{i, j} a_{i j} \partial_{i} \partial_{j}+\sum_{i} b_{i} \partial_{i}$ on $\mathbb{R}^{d}$. It is proved that there exists $c \in \mathbb{R}$ and an $\mathbb{R}^{d}$-valued function $b=\left(b_{i}\right)$ such that $\left|\nabla P_{t} f\right| \leq \mathrm{e}^{c t} P_{t}|\nabla f|$ holds for all $t>0$ and all $f \in C_{b}^{1}\left(\mathbb{R}^{d}\right)$ if and only if $a=\left(a_{i j}\right)$ satisfies the formula $\partial_{k} a_{i j}+\partial_{j} a_{k i}+\partial_{i} a_{k j}=0$ for all $i, j, k$.
\end{abstract}

\section{INTRODUCTION}

Consider the second-order differential operator on $\mathbb{R}^{d}$ :

$$
L=L_{a, b}:=\sum_{i, j=1}^{d} a_{i j} \partial_{i} \partial_{j}+\sum_{i=1}^{d} b_{i} \partial_{i},
$$

where $a=\sigma \sigma^{*}$ with $\sigma$ a matrix-valued $C^{1}$-function such that $a$ is non-degenerate, and $b$ an $\mathbb{R}^{d}$-valued $C^{1}$-function. Assume that the $L$-diffusion process is nonexplosive and let $P_{t}$ denote its semigroup. The main purpose of the paper is to search for explicit criteria for the following gradient estimate of $P_{t}$ :

$$
\left|\nabla P_{t} f\right| \leq \mathrm{e}^{c t} P_{t}|\nabla f|, \quad t \geq 0, f \in C_{b}^{1}\left(\mathbb{R}^{d}\right),
$$

where $c \in \mathbb{R}$ is a constant, and $\nabla$ and $|\cdot|$ are, respectively, the Euclidean gradient operator and the Euclidean norm.

The gradient estimates of diffusion semigroups have played an important role in the study of functional inequalities and heat kernels. For instance, (1.1) has been related to (at least for $a \equiv I$ ) the log-Sobolev inequality, the dimension-free Harnack inequality and heat kernel upper bounds (see e.g. [2, 6, 7, 8, 5]). Indeed, (1.2) with negative $c$ can imply the log-Sobolev inequality even if $a$ is not constant (cf. [4]).

To search for the exact and simple feature of (1.1), we make use of the following equivalent condition following the line of Bakry [1]:

$$
\langle\nabla f, \nabla L f\rangle \leq|\nabla f| L|\nabla f|+c|\nabla f|^{2}, \quad f \in C^{\infty}\left(\mathbb{R}^{d}\right),
$$

Received by the editors February 6, 2002 and, in revised form, November 15, 2003.

2000 Mathematics Subject Classification. Primary 47D07, 60H10.

This work was supported in part by NNSFC(10025105, 10121101), TRAPOYT in China and the 973-Project. 
where we set $|\nabla f| L|\nabla f|=0$ when $|\nabla f|=0$. Starting from this equivalence, we obtain with some efforts the following result which implies that the formula (1.3) below is the character of (1.1).

Theorem 1.1. (1) (1.1) implies

$$
\partial_{k} a_{i j}+\partial_{i} a_{k j}+\partial_{j} a_{k i}=0, \quad 1 \leq i, j, k \leq d .
$$

(2) If (1.3) holds, then for any $c \in \mathbb{R}$ there exists b such that (1.1) holds for the diffusion semigroup generated by $L_{a, b}$. More precisely, (1.1) follows from (1.3) and

$$
c \geq \sup \left\{\frac{1}{2 \lambda(a)(x)} \sum_{i, j=1}^{d}\left|\partial_{X} a_{i j}\right|^{2}(x)+\left\langle\partial_{X} b, X\right\rangle(x): x, X \in \mathbb{R}^{d},|X|=1\right\} .
$$

When $a \equiv I$, according to Bakry [1], (1.1) is equivalent to

$$
\left|\nabla P_{t} f\right|^{2} \leq \mathrm{e}^{2 c t} P_{t}|\nabla f|^{2}, \quad t \geq 0, f \in C_{b}^{1}\left(\mathbb{R}^{d}\right) .
$$

It is easy to see from [1] that (1.5) is always equivalent to

$$
\langle\nabla f, \nabla L f\rangle \leq \frac{1}{2} L|\nabla f|^{2}+c|\nabla f|^{2}, \quad f \in C^{\infty}\left(\mathbb{R}^{d}\right) .
$$

But by combining the following result with Theorem 1.1 we see that 1.1 is not equivalent to (1.5) in general, since it is easy to find an example such that (1.7) holds but (1.3) does not hold (e.g. $\left.d=1, a(x)=\left(1+x^{2}\right)^{1 / 2}, b=0\right)$.

Proposition 1.2. Let $\lambda(a)(x)$ denote the minimal eigenvalue of a $(x)$ for each $x$. (1.5) holds provided

$$
c \geq \sup \left\{\frac{1}{4 \lambda(a)(x)} \sum_{i, j=1}^{d}\left|\partial_{X} a_{i j}\right|^{2}(x)+\left\langle\partial_{X} b, X\right\rangle(x): x, X \in \mathbb{R}^{d},|X|=1\right\} .
$$

Moreover, to see that (1.7) is somehow a reasonable condition for (1.5) to hold, we present the following estimate of $\left(\partial_{X} a_{i j}\right)^{2}$ in terms of $b$ and $c$.

Proposition 1.3. If (1.5) holds, then $\left\langle\partial_{X} b, X\right\rangle \leq c|X|^{2}$ for all $X$, and

$$
\sum_{i, j=1}^{d}\left(\partial_{X} a_{i j}\right)^{2} \leq 4 \bar{\lambda}(a)\left(c-\left\langle\partial_{X} b, X\right\rangle\right), \quad|X|=1
$$

where at each point $\bar{\lambda}(a)$ denotes the maximal eigenvalue of a.

Remark 1.1. In general, (1.1) holds provided (see the proof of Theorem 4.13 in [3] )

$$
\operatorname{Tr}\left\{(\sigma(x)-\sigma(y))\left(\sigma(x)^{*}-\sigma(y)^{*}\right)\right\}+\langle b(x)-b(y), x-y\rangle \leq c|x-y|^{2}, \quad x, y \in \mathbb{R}^{d} .
$$

Recently, Da Prato and Goldys claimed in [4] that (1.9) also implies (1.1) provided $a \geq \varepsilon I$ for some $\varepsilon>0$. This assertion, however, is unfortunately wrong: there are a lot of counter-examples according to Theorem 1.1. Indeed, there is an obvious gap in their proof, i.e. in the proof of Proposition 2.8 in [4]: what they obtained is an upper bound of the second moment, rather than the uniform norm as they claimed, of the derivative process; thus, what they could prove there is (1.5) rather than (1.1). 


\section{Proofs}

We first present the following simple lemma, which essentially follows from the standard argument of Bakry (cf. [1]). A complete proof is presented as an appendix for the reader's reference.

Lemma 2.1. (1.1) is equivalent to (1.2), while (1.5) is equivalent to (1.6).

Proof of Theorem 1.1 (1). We shall only prove (1.3) at the point 0 , since at other points the proof also works by shifting our test functions. We consider three cases respectively: $i=j=k ; i \neq j$ but $k \in\{i, j\}$; and different $i, j, k$.

(a) For any $i$ one has $\partial_{i} a_{i i}=0$. Let $f(x)=\cos x_{i}$. By (1.2), we have

$$
\partial_{i} a_{i i}(x) \leq\left(c-\partial_{i} b_{i}(x)\right) \tan x_{i}, \quad x_{i}>0 .
$$

Letting $x \rightarrow 0$ with $x_{i}>0$, we obtain $\partial_{i} a_{i i}(0) \leq 0$. Similarly, for $x_{i}<0$ one has $\partial_{i} a_{i i}(x) \geq\left(c-\partial_{i} b_{i}(x)\right) \tan x_{i}$, and hence $\partial_{i} a_{i i}(0) \geq 0$.

(b) For $i \neq j$, it holds that $2 \partial_{i} a_{i j}+\partial_{j} a_{i i}=0$. For $\varepsilon>0$, let $f(x)=\left(\varepsilon x_{j}+x_{i}\right)^{2}$. For $x_{i}, x_{j}>0$ we have

$$
|\nabla f|(x)=2\left(\varepsilon x_{j}+x_{i}\right) \sqrt{1+\varepsilon^{2}}, \quad|\nabla f| L|\nabla f|=4\left(1+\varepsilon^{2}\right)\left(\varepsilon x_{j}+x_{i}\right)\left(\varepsilon b_{j}+b_{i}\right) .
$$

Moreover, since $\partial_{i} a_{i i}=\partial_{j} a_{j j}=0$, we have

$$
\begin{aligned}
\langle\nabla f, \nabla L f\rangle= & 4\left(\varepsilon x_{j}+x_{i}\right)\left(\varepsilon \partial_{j}+\partial_{i}\right)\left[a_{i i}+\varepsilon^{2} a_{j j}+2 \varepsilon a_{i j}+\left(\varepsilon x_{j}+x_{i}\right)\left(\varepsilon b_{j}+b_{i}\right)\right] \\
= & 4\left(\varepsilon x_{j}+x_{i}\right)\left(\varepsilon \partial_{j} a_{i i}+2 \varepsilon^{2} \partial_{j} a_{i j}+\varepsilon^{2} \partial_{i} a_{j j}+2 \varepsilon \partial_{i} a_{i j}\right) \\
& +4\left(\varepsilon x_{j}+x_{i}\right)\left(\varepsilon b_{j}+b_{i}\right)\left(1+\varepsilon^{2}\right)+4\left(\varepsilon x_{j}+x_{i}\right)^{2}\left(\varepsilon \partial_{j}+\partial_{i}\right)\left(\varepsilon b_{j}+b_{i}\right) .
\end{aligned}
$$

Therefore, by (1.2),

$$
2 \partial_{i} a_{i j}+\partial_{j} a_{i i} \leq \frac{\varepsilon x_{j}+x_{i}}{\varepsilon}\left[c\left(1+\varepsilon^{2}\right)-\left(\varepsilon \partial_{j}+\partial_{i}\right)\left(\varepsilon b_{j}+b_{i}\right)\right]-\varepsilon\left(2 \partial_{j} a_{i j}+\partial_{i} a_{j j}\right) .
$$

Letting $x \rightarrow 0$ with $x_{i}, x_{j}>0$, we obtain

$$
\left(2 \partial_{i} a_{i j}+\partial_{j} a_{j j}\right)(0) \leq-\varepsilon\left(2 \partial_{j} a_{i j}+\partial_{i} a_{i j}\right)(0) .
$$

Thus, letting $\varepsilon \downarrow 0$ we arrive at $\left(2 \partial_{i} a_{i j}+\partial_{j} a_{i i}\right)(0) \leq 0$. Similarly, working with $x_{i}, x_{j}<0$ we obtain the inverse inequality.

(c) For any different $i, j, k$, one has $\partial_{k} a_{i j}+\partial_{i} a_{k j}+\partial_{j} a_{i k}=0$. Let $f(x)=$ $\left(x_{k}+x_{i}+x_{j}\right)^{2}$. For $x_{i}, x_{j}, x_{k}>0$ we have

$$
\begin{aligned}
& |\nabla f(x)|=2 \sqrt{3}\left(x_{k}+x_{i}+x_{j}\right):=2 \sqrt{3} g(x), \\
& (|\nabla f| L|\nabla f|)(x)=12 g(x)\left(b_{k}+b_{i}+b_{j}\right)(x) .
\end{aligned}
$$

Moreover, by (a) and (b) in this proof we have

$\langle\nabla f, \nabla L f\rangle$

$$
\begin{aligned}
= & 4 g \cdot\left(\partial_{k}+\partial_{i}+\partial_{j}\right)\left[a_{i i}+a_{j j}+a_{k k}+2 a_{i j}+2 a_{k i}+2 a_{k j}+g \cdot\left(b_{k}+b_{i}+b_{j}\right)\right] \\
= & 4 g^{2} \cdot\left(\partial_{k}+\partial_{i}+\partial_{j}\right)\left(b_{k}+b_{i}+b_{j}\right)+12 g \cdot\left(b_{k}+b_{i}+b_{j}\right) \\
& +8 g \cdot\left(\partial_{k} a_{i j}+\partial_{j} a_{i k}+\partial_{i} a_{j k}\right) .
\end{aligned}
$$

Therefore, for $x_{i}, x_{j}, x_{k}>0$ it follows from (1.2) that

$$
2\left(\partial_{k} a_{i j}+\partial_{j} a_{i k}+\partial_{i} a_{j k}\right) \leq 3 c g-g \cdot\left(\partial_{k}+\partial_{i}+\partial_{j}\right)\left(b_{k}+b_{i}+b_{j}\right) .
$$

Letting $x \rightarrow 0$ we obtain $\left(\partial_{k} a_{i j}+\partial_{j} a_{i k}+\partial_{i} a_{j k}\right)(0) \leq 0$. Similarly, we obtain the inverse inequality by considering $x_{i}, x_{j}, x_{k}<0$. 
Proof of Theorem 1.1 (2). We first prove that (1.4) and (1.3) imply (1.2). For $f \in C_{0}^{\infty}\left(\mathbb{R}^{d}\right)$ we have

$$
\langle a \nabla|\nabla f|, \nabla|\nabla f|\rangle=\frac{1}{|\nabla f|^{2}} \sum_{i, j=1}^{d} a_{i j}\left\langle\nabla f, \nabla f_{i}^{\prime}\right\rangle\left\langle\nabla f, \nabla f_{j}^{\prime}\right\rangle
$$

at points with $|\nabla f|>0$. Moreover, we have

$$
\langle\nabla f, \nabla L f\rangle=\sum_{i, j=1}^{d}\left[\left\langle\nabla f, \nabla a_{i j}\right\rangle f_{i j}^{\prime \prime}+a_{i j}\left\langle\nabla f, \nabla f_{i j}^{\prime \prime}\right\rangle\right]+\sum_{i=1}^{d}\left[b_{i}\left\langle\nabla f, \nabla f_{i}^{\prime}\right\rangle+f_{i}^{\prime}\left\langle\nabla f, \nabla b_{i}\right\rangle\right],
$$

$$
\frac{1}{2} L|\nabla f|^{2}=\sum_{i, j=1}^{d} a_{i j}\left[\left\langle\nabla f_{i}^{\prime}, \nabla f_{j}^{\prime}\right\rangle+\left\langle\nabla f, \nabla f_{i j}^{\prime \prime}\right\rangle\right]+\sum_{i=1}^{d} b_{i}\left\langle\nabla f, \nabla f_{i}^{\prime}\right\rangle,
$$

where $f_{j}^{\prime}:=\partial_{j} f$ and $f_{i j}^{\prime \prime}:=\partial_{i} \partial_{j} f, 1 \leq i, j \leq d$. Combining (2.1) with (2.2) and (2.3) we obtain

$$
\begin{aligned}
I_{1} & :=\langle\nabla f, \nabla L f\rangle-|\nabla f| L|\nabla f|=\langle\nabla f, \nabla L f\rangle-\frac{1}{2} L|\nabla f|^{2}+\langle a \nabla|\nabla f|, \nabla|\nabla f|\rangle \\
& =\sum_{i, j=1}^{d}\left\{\left\langle\nabla f, \nabla a_{i j}\right\rangle f_{i j}^{\prime \prime}-a_{i j}\left(\left\langle\nabla f_{i}^{\prime}, \nabla f_{j}^{\prime}\right\rangle-\frac{\left\langle\nabla f, \nabla f_{i}^{\prime}\right\rangle\left\langle\nabla f, \nabla f_{j}^{\prime}\right\rangle}{|\nabla f|^{2}}\right)\right\}+\left\langle\partial_{\nabla f} b, \nabla f\right\rangle \\
& =\sum_{i, j=1}^{d}\left\{\left\langle\nabla f, \nabla a_{i j}\right\rangle f_{i j}^{\prime \prime}-a_{i j}\left\langle P\left(\nabla f_{i}^{\prime}\right), P\left(\nabla f_{j}^{\prime}\right)\right\rangle\right\}+\left\langle\partial_{\nabla f} b, \nabla f\right\rangle,
\end{aligned}
$$

where $P$ denotes the projection to the vector space $\{X:\langle X, \nabla f\rangle=0\}$. To prove (1.5) we need to estimate

$$
I_{2}:=\sum_{i, j=1}^{d}\left\langle\nabla f, \nabla a_{i j}\right\rangle f_{i j}^{\prime \prime}=\sum_{i, j, k=1}^{d} f_{k}^{\prime}\left(\partial_{k} a_{i j}\right) f_{i j}^{\prime \prime}
$$

in terms of $\sum_{i}\left|P\left(\nabla f_{i}^{\prime}\right)\right|^{2}$. To this end, let us look at a fixed point $x_{0}$ with $|\nabla f|\left(x_{0}\right)>$ 0 . Since $I_{1}$ involves $a$ only via $a_{i j}\left(x_{0}\right)$ and $\nabla a_{i j}\left(x_{0}\right)$, we may assume that $a_{i j}$ is linear for all $i, j$. Indeed, replacing $a_{i j}(x)$ by $a_{i j}\left(x_{0}\right)+\sum_{k} x_{k} \partial_{k} a_{i j}\left(x_{0}\right)$, the value of $I_{1}$ at $x_{0}$ does not change and (1.3) remains true.

Now, for each $j$ let $X^{j}$ be an $\mathbb{R}^{d}$-valued function with $X_{i}^{j}:=\sum_{k=1}^{d} f_{k}^{\prime} \partial_{k} a_{i j}, 1 \leq$ $i \leq d$. We have

$$
I_{2}=\sum_{j=1}^{d}\left\langle\nabla f_{j}^{\prime}, X^{j}\right\rangle=\sum_{j=1}^{d}\left\langle P\left(X^{j}\right), P\left(\nabla f_{j}^{\prime}\right)\right\rangle+\frac{1}{|\nabla f|^{2}} \sum_{j=1}^{d}\left\langle\nabla f_{j}^{\prime}, \nabla f\right\rangle\left\langle\nabla f, X^{j}\right\rangle .
$$

Since by (1.3) one has

$$
\sum_{j=1}^{d}\left\langle X^{j}, \nabla f\right\rangle f_{j}^{\prime}=\sum_{i, j, k=1}^{d} f_{i}^{\prime} f_{j}^{\prime} f_{k}^{\prime} \partial_{k} a_{i j}=0
$$

it follows that

$$
0=\sum_{j=1}^{d}\left\langle\nabla f, \nabla\left(\left\langle X^{j}, \nabla f\right\rangle f_{j}^{\prime}\right)\right\rangle=\sum_{j=1}^{d}\left[\left\langle\nabla f_{j}^{\prime}, \nabla f\right\rangle\left\langle X^{j}, \nabla f\right\rangle+f_{j}^{\prime}\left\langle\nabla f, \nabla\left\langle X^{j}, \nabla f\right\rangle\right\rangle\right] .
$$


Noting that we have assumed that $a_{i j}$ is linear for each $i, j$, we obtain

$$
\begin{aligned}
I_{3} & :=\sum_{j=1}^{d}\left\langle\nabla f_{j}^{\prime}, \nabla f\right\rangle\left\langle\nabla f, X^{j}\right\rangle=-\sum_{j, k=1}^{d} f_{j}^{\prime} f_{k}^{\prime} \partial_{k}\left(\sum_{i=1}^{d} f_{i}^{\prime} X_{i}^{j}\right) \\
& =-\sum_{i, j, k, l=1}^{d} f_{j}^{\prime} f_{k}^{\prime}\left[f_{i} f_{k l}^{\prime \prime} \partial_{l} a_{i j}+f_{l}^{\prime} f_{i k}^{\prime \prime} \partial_{l} a_{i j}\right] .
\end{aligned}
$$

Combining this with (1.3) we arrive at

$$
I_{3}=-\sum_{i, j, k, l=1}^{d} f_{j}^{\prime} f_{k}^{\prime}\left[f_{i}^{\prime} f_{k l}^{\prime \prime} \partial_{l} a_{i j}-f_{l}^{\prime} f_{i k}^{\prime \prime} \partial_{i} a_{l j}-f_{l}^{\prime} f_{i k}^{\prime \prime} \partial_{j} a_{i l}\right]=\sum_{i, j, k, l=1}^{d} f_{j}^{\prime} f_{k}^{\prime} f_{l}^{\prime} f_{i k}^{\prime \prime} \partial_{j} a_{i l} .
$$

For fixed $k$, let $Y^{k}$ be the $\mathbb{R}^{d}$-valued function with $Y_{i}^{k}:=f_{k}^{\prime} \sum_{j, l=1}^{d} f_{j}^{\prime} f_{l}^{\prime} \partial_{j} a_{i l}$. By (2.6) we have

$$
\left\langle Y^{k}, \nabla f\right\rangle=f_{k}^{\prime} \sum_{i, j, l=1}^{d} f_{i}^{\prime} f_{j}^{\prime} f_{l}^{\prime} \partial_{j} a_{i l}=0
$$

Then

$$
I_{3}=\sum_{k=1}^{d}\left\langle Y^{k}, \nabla f_{k}^{\prime}\right\rangle=\sum_{k=1}^{d}\left\langle Y^{k}, P\left(\nabla f_{k}^{\prime}\right)\right\rangle
$$

Combining this with (2.4) and (2.5) we obtain

$$
\begin{aligned}
I_{1}= & \sum_{j=1}^{d}\left(\left\langle P\left(X^{j}\right), P\left(\nabla f_{j}^{\prime}\right)\right\rangle+\frac{1}{|\nabla f|^{2}}\left\langle Y^{j}, P\left(\nabla f_{j}^{\prime}\right)\right\rangle\right) \\
& -\sum_{i, j=1}^{d} a_{i j}\left\langle P\left(\nabla f_{i}^{\prime}\right), P\left(\nabla f_{j}^{\prime}\right)\right\rangle+\left\langle\partial_{\nabla f} b, \nabla f\right\rangle \\
\leq & |\nabla f|\left\{\sum_{j=1}^{d}\left|P\left(\nabla f_{j}^{\prime}\right)\right|^{2}\right\}^{1 / 2}\left\{\frac{2}{|\nabla f|^{2}} \sum_{j=1}^{d}\left|P\left(X^{j}\right)\right|^{2}+\frac{2}{|\nabla f|^{6}} \sum_{j=1}^{d}\left|Y^{j}\right|^{2}\right\}^{1 / 2} \\
& \quad-\lambda(a) \sum_{j=1}^{d}\left|P\left(\nabla f_{j}^{\prime}\right)\right|^{2}+\left\langle\partial_{\nabla f} b, \nabla f\right\rangle \\
\leq & \frac{|\nabla f|^{2}}{2 \lambda(a)} \sum_{j=1}^{d}\left(\frac{\left|P\left(X^{j}\right)\right|^{2}}{|\nabla f|^{2}}+\frac{\left|Y^{j}\right|^{2}}{|\nabla f|^{6}}\right)+\left\langle\partial_{\nabla f} b, \nabla f\right\rangle .
\end{aligned}
$$

Since

$$
\sum_{j=1}^{d}\left|X^{j}\right|^{2}=\sum_{i, j=1}^{d}\left(\sum_{k=1}^{d} f_{k}^{\prime} \partial_{k} a_{i j}\right)^{2}=\sum_{i, j=1}^{d}\left(\partial_{\nabla f} a_{i j}\right)^{2}
$$

by (2.7), (1.2) follows from

$$
\sum_{j=1}^{d}\left(\left|P\left(X^{j}\right)\right|^{2}+\frac{\left|Y^{j}\right|^{2}}{|\nabla f|^{4}}\right)=\sum_{j=1}^{d}\left|X^{j}\right|^{2}
$$


or equivalently

$$
|\nabla f|^{2} \sum_{j=1}^{d}\left\langle X^{j}, \nabla f\right\rangle^{2}=\sum_{j=1}^{d}\left|Y^{j}\right|^{2} .
$$

By the definitions of $X^{j}$ and $Y^{k}$, we have

$$
\begin{aligned}
\sum_{k=1}^{d}\left|Y^{k}\right|^{2} & =|\nabla f|^{2} \sum_{i=1}^{d}\left(\sum_{j, l=1}^{d} f_{j}^{\prime} f_{l}^{\prime} \partial_{j} a_{i l}\right)^{2}=|\nabla f|^{2} \sum_{j=1}^{d}\left(\sum_{i, l=1}^{d} f_{i}^{\prime} f_{l}^{\prime} \partial_{i} a_{j l}\right)^{2} \\
& =|\nabla f|^{2} \sum_{j=1}^{d}\left(\sum_{i, l=1}^{d} f_{i}^{\prime} f_{l}^{\prime} \partial_{l} a_{i j}\right)^{2}=|\nabla f|^{2} \sum_{j=1}^{d}\left\langle X^{j}, \nabla f\right\rangle^{2} .
\end{aligned}
$$

Thus (2.8) and hence (1.2) holds.

Now, assume that (1.3) holds and let $c \in \mathbb{R}$ be fixed. Let $F \in C^{2}([0, \infty))$ be such that $F^{\prime} \geq 0$ and

$$
F\left(|x|^{2}\right) \geq \frac{1}{2 \lambda(a)(x)} \sum_{i, j=1}^{d}\left|\nabla a_{i j}(x)\right|^{2}, \quad x \in \mathbb{R}^{d} .
$$

Indeed, letting $\gamma \in C^{2}[0, \infty)$ with

$$
\gamma(r) \geq \sup _{|x|^{2}=r} \frac{1}{2 \lambda(a)(x)} \sum_{i, j=1}^{d}\left|\nabla a_{i j}(x)\right|^{2},
$$

we may take $F(r)=\gamma(0)+\int_{0}^{r} \sqrt{1+\gamma^{\prime}(s)^{2}} \mathrm{~d} s$. Let

$$
V(x)=-\frac{1}{2} \int_{0}^{|x|^{2}} F(r) \mathrm{d} r+\frac{1}{2} c|x|^{2}, \quad b(x)=\nabla V(x) .
$$

We have

$$
\left\langle\partial_{X} b, X\right\rangle(x)=\operatorname{Hess}_{V}(X, X)(x) \leq \frac{1}{2}\left(c-F\left(|x|^{2}\right)\right) \operatorname{Hess}|\cdot|^{2}(X, X)(x)=c-F\left(|x|^{2}\right) .
$$

Therefore, (1.4) holds and hence one has (1.1).

Proof of Proposition 1.2. By Lemma 2.1, it suffices to show that each of (1.7) implies (1.6). By (2.2) and (2.3) we have

$$
\begin{aligned}
\langle\nabla f, \nabla L f\rangle-\frac{1}{2} L|\nabla f|^{2} & =\sum_{i, j=1}^{d}\left\langle\nabla f, \nabla a_{i j}\right\rangle f_{i j}^{\prime \prime}-\sum_{i, j=1}^{d} a_{i j}\left\langle\nabla f_{i}^{\prime}, \nabla f_{j}^{\prime}\right\rangle+\left\langle\partial_{\nabla f} b, \nabla f\right\rangle \\
& \leq \sum_{i, j=1}^{d}\left\langle\nabla f, \nabla a_{i j}\right\rangle f_{i j}^{\prime \prime}-\lambda(a) \sum_{i, j=1}^{d}\left(f_{i j}^{\prime \prime}\right)^{2}+\left\langle\partial_{\nabla f} b, \nabla f\right\rangle \\
& \leq \frac{1}{4 \lambda(a)} \sum_{i, j=1}^{d}\left|\partial_{\nabla f} a_{i j}\right|^{2}+\left\langle\partial_{\nabla f} b, \nabla f\right\rangle .
\end{aligned}
$$

Thus (1.7) implies (1.6) and hence (1.5) by Lemma 2.1 . 
Proof of Proposition 1.3. The estimate of $\left\langle\partial_{X} b, X\right\rangle$ follows from (1.6) with $f(x)=$ $\sum_{i, j} X_{i} x_{i}$. Now, for fixed $X, x \in \mathbb{R}^{d}$ with $|X|=1$, let

$$
f(y)=2 \bar{\lambda}(a)(x)\langle X, y\rangle+\frac{1}{2} \sum_{i, j=1}^{d}\left(\partial_{X} a_{i j}(x)\right)\left(y_{i}-x_{i}\right)\left(y_{j}-x_{j}\right) .
$$

It follows from (1.6), (2.2) and (2.3) that

$$
\begin{aligned}
4 c \bar{\lambda}(a)^{2}(x) & =c|\nabla f(x)|^{2} \geq\langle\nabla f, \nabla L f\rangle(x)-\frac{1}{2} L|\nabla f|^{2}(x) \\
& =\sum_{i, j=1}^{d}\left[\left\langle\nabla f, \nabla a_{i j}\right\rangle f_{i j}^{\prime \prime}-a_{i j}\left\langle\nabla f_{i}^{\prime}, \nabla f_{j}^{\prime}\right\rangle\right](x)+\langle\nabla \nabla f b, \nabla f\rangle(x) \\
& \geq \bar{\lambda}(a)(x) \sum_{i, j=1}^{d}\left(\partial_{X} a_{i j}(x)\right)^{2}+4 \bar{\lambda}(a)^{2}(x)\left\langle\partial_{X} b(x), X\right\rangle^{2} .
\end{aligned}
$$

This implies (1.8).

\section{Appendix: the PRoof of Lemma 2.1}

The basic idea of the proof comes from Bakry [1]. To follow his argument, we need to show that $\left|\nabla P_{s} f\right|^{2}$ is in the weak domain of $L$ for $f \in C_{0}^{\infty}\left(\mathbb{R}^{d}\right)$ and $s>0$, for which we make use of Itô's formula. We only prove the equivalence of (1.1) and (1.2) since that of (1.5) and (1.6) is even simpler.

(a) (1.1) implies (1.2). We only prove the second implication since the proof of the first is similar and simpler. Assume (1.1); it suffices to show (1.2) for $f \in C_{0}^{\infty}\left(\mathbb{R}^{d}\right)$ at points with $|\nabla f|>0$. Let $x \in \mathbb{R}^{d}$ be such that $|\nabla f|(x)>0$, take $g \in C_{0}^{\infty}\left(\mathbb{R}^{d}\right)$ such that $g \geq|\nabla f|$ on $\mathbb{R}^{d}$ and $g=|\nabla f|$ in a neighborhood of $x$. Then it follows from (1.1) that

$$
\left|\nabla P_{t} f\right|^{2} \leq \mathrm{e}^{2 c t}\left(P_{t} g\right)^{2}
$$

for all $t \geq 0$ with equality holding at $t=0$. Therefore, we are able to take derivatives of both sides w.r.t. $t$ at $t=0$ to obtain

$$
2\langle\nabla f, \nabla L f\rangle(x) \leq 2\left(c g^{2}+g L g\right)(x)=2\left(c|\nabla f|^{2}+|\nabla f| L|\nabla f|\right)(x) .
$$

(b) (1.2) implies (1.1). Let $t>0$ and $f \in C_{0}^{\infty}\left(\mathbb{R}^{d}\right)$ be fixed. For $\delta>0$ let

$$
h_{\delta}(r)=\frac{2}{\delta^{2}} \int_{0}^{r} \mathrm{~d} s \int_{0}^{s}(\delta-t)^{+} \mathrm{d} t, \quad r \geq 0 .
$$

Then $h_{\delta} \in C^{2}[0, \infty)$ with $h_{\delta}^{\prime}(0)=0,0 \leq h_{\delta}^{\prime} \leq 1,0 \leq h_{\delta}^{\prime \prime}$ and $h_{\delta}(r) \uparrow r, h_{\delta}^{\prime}(r) \uparrow 1$ for $r>0$ as $\delta \downarrow 0$. Let $\left\{x_{s}\right\}$ denote the $L$-diffusion process and let $\tau_{n}:=\inf \{s \in[0, t]$ : $\left.\left|x_{t}\right| \geq n\right\}$ where we set $\inf \emptyset=t$. Then there is a martingale $\left\{M_{s}\right\}$ on $\left[0, \tau_{n}\right]$ such that

$$
\begin{aligned}
\mathrm{d} h_{\delta}(\mid & \left.\nabla P_{\tau_{n}-s} f \mid\right)\left(x_{s}\right) \\
=\quad \mathrm{d} M_{s}+L h_{\delta}\left(\left|\nabla P_{\tau_{n}-s} f\right|\right)\left(x_{s}\right) \mathrm{d} s & \\
& -\left\{\frac{h_{\delta}^{\prime}\left(\left|\nabla P_{\tau_{n}-s} f\right|\right)}{\left|\nabla P_{\tau_{n}-s} f\right|}\left\langle\nabla P_{\tau_{n}-s} f, \nabla L P_{\tau_{n}-s} f\right\rangle\right\}\left(x_{s}\right) \mathrm{d} s, \quad s \in\left[0, \tau_{n}\right] .
\end{aligned}
$$

Since $h_{\delta}^{\prime \prime} \geq 0$, combining this with (1.2) we obtain

$$
\mathrm{d} h_{\delta}\left(\left|\nabla P_{\tau_{n}-s} f\right|\right)\left(x_{s}\right) \geq \mathrm{d} M_{s}-c\left\{h_{\delta}^{\prime}\left(\left|\nabla P_{\tau_{n}-s} f\right|\right)\left|\nabla P_{\tau_{n}-s} f\right|\right\}\left(x_{s}\right) \mathrm{d} s, \quad s \in\left[0, \tau_{n}\right] .
$$


Therefore,

$$
\begin{aligned}
& E h_{\delta}\left(\left|\nabla P_{\tau_{n}-s \wedge \tau_{n}} f\right|\right)\left(x_{s \wedge \tau_{n}}\right)-E h_{\delta}\left(\left|\nabla P_{\tau_{n}-s^{\prime} \wedge \tau_{n}} f\right|\right)\left(x_{s^{\prime} \wedge \tau_{n}}\right) \\
& \quad \geq-c E \int_{s^{\prime} \wedge \tau_{n}}^{s \wedge \tau_{n}}\left(h_{\delta}^{\prime}\left(\left|\nabla P_{\tau_{n}-u} f\right|\right)\left|\nabla P_{\tau_{n} t-u} f\right|\right)\left(x_{u}\right) \mathrm{d} u, \quad 0 \leq s^{\prime}<s \leq t .
\end{aligned}
$$

Let $\phi_{n}(s):=E\left|\nabla P_{\tau_{n}-s \wedge \tau_{n}} f\right|\left(x_{s \wedge \tau_{n}}\right)$. By letting $\delta \downarrow 0$ in the above inequality, we obtain

$$
\phi_{n}(s)-\phi_{n}\left(s^{\prime}\right) \geq-c E \int_{s^{\prime} \wedge \tau_{n}}^{s \wedge \tau_{n}}\left|\nabla P_{\tau_{n}-u} f\right|\left(x_{u}\right) \mathrm{d} u \geq-c^{+} \int_{s^{\prime}}^{s} \phi_{n}(u) \mathrm{d} u, \quad t \geq s>s^{\prime} \geq 0 .
$$

Thus $\phi_{n}(t) \geq \mathrm{e}^{-c^{+} t} \phi_{n}(0)$. Since $|\nabla f|$ is bounded, by Fatou's lemma and the dominated convergence theorem we obtain

$$
\left|\nabla P_{t} f\right|=E \liminf _{n \rightarrow \infty}\left|\nabla P_{\tau_{n}} f\right| \leq \liminf _{n \rightarrow \infty} \phi_{n}(0) \leq \mathrm{e}^{c^{+} t} \liminf _{n \rightarrow \infty} \phi_{n}(t)=\mathrm{e}^{c^{+} t} P_{t}|\nabla f| .
$$

Therefore, we are able to use the dominated convergence theorem by letting $n \rightarrow \infty$ in the first inequality of (3.1) to obtain

$$
E\left|\nabla P_{t-s} f\right|\left(x_{s}\right)-E\left|\nabla P_{t-s^{\prime}} f\right|\left(x_{s^{\prime}}\right) \geq-c \int_{s^{\prime}}^{s} E\left|\nabla P_{t-u} f\right|\left(x_{u}\right) \mathrm{d} u .
$$

This implies (1.1).

\section{ACKNOWLEDGMENT}

The work was initiated when the author visited Bielefeld during the summer of 2001. He would like to thank Professor M. Röckner for warm hospitality and useful discussions.

\section{REFERENCES}

[1] D. Bakry, On Sobolev and logarithmic Sobolev inequalities for Markov semigroups, New Trends in Stochastic Analysis (Eds. K.D. Elworthy, S. Kusuoka, I. Shigekawa), 43-75, World Scientific, 1997. MF 99m:60110

[2] D. Bakry and M. Ledoux, Lévy-Gromov's isoperimetric inequality for an infinite-dimensional diffusion generator, Invent. Math. 123(1996), 253-270. MR97c:58162

[3] M. F. Chen and F. Y. Wang, Estimation of spectral gap for elliptic operators, Trans. Amer. Math. Soc. 349(1997), 1239-1267. MR.97h:35175

[4] G. Da Prato and B. Goldys, Elliptic operators on $\mathbb{R}^{d}$ with unbounded coefficients, J. Diff. Equat. 172 (2001), 333-358. MR2002d:35228

[5] F.-Z. Gong and F.-Y. Wang, Heat kernel estimates with application to compactness of manifolds, Quart. J. Math. 52(2001), 171-180. MR.2002c:58039

[6] E. P. Hsu, Logarithmic Sobolev inequalities on path spaces over Riemannian manifolds, Comm. Math. Phys. 189(1997), 9-16. MR98i:58249

[7] F.-Y. Wang, Logarithmic Sobolev inequalities on noncompact Riemannian manifolds, Probab. Theory Relat. Fields 109(1997), 417-424. MR98i:58253

[8] F.-Y. Wang, Functional inequalities, semigroup properties and spectrum estimates, Inf. Dimens. Anal. Quant. Probab. Relat. Topics 3(2000), 263-295. MR2002b:47083

Department of Mathematics, Beijing Normal University, Beijing 100875, People's Republic of China

E-mail address: wangfy@bnu.edu.cn 